\title{
PSEUDO ASYMPTOTICALLY PERIODIC SOLUTIONS OF TWO-TERM TIME FRACTIONAL DIFFERENTIAL EQUATIONS WITH DELAY
}

\author{
ZHINAN XIA
}

\begin{abstract}
In this paper, the existence and uniqueness of pseudo $\mathcal{S}$-asymptotically $\omega$-periodic mild solutions of class $r$ for the nonlinear two-term time fractional differential equations with delay are investigated. The working tools are based on the generalization of the semigroup theory and fixed point theory. Finally, we present an application to a fractional partial differential equation with delay.
\end{abstract}

\section{Introduction}

The study of the existence of asymptotically $\omega$-periodic solutions is one of the most interesting and important topics in the qualitative theory of differential equations. From an applied perspective, asymptotically $\omega$-periodic systems describe world more realistically and accurately than periodic ones, one can see $[7,12,13,22,23]$ for more details. The notion of $\mathcal{S}$-asymptotic $\omega$-periodicity, introduced by Henríquez et al. in $[10,11]$, is related to and more general than that of asymptotic $\omega$-periodicity. Since then, it has attracted the attention of many researchers and the interest in this topic still increases [3, 4, 5, 8, 9, 16, 18]. Recently, in [19], the concept of pseudo $\mathcal{S}$-asymptotic $\omega$-periodicity and pseudo $\mathcal{S}$ asymptotic $\omega$-periodicity of class $r$, which generalizes the notion of $\mathcal{S}$-asymptotic $\omega$-periodicity, was introduced and the applications to semilinear first-order differential equations with delay in Banach spaces were studied.

In this paper, we study the existence, uniqueness of pseudo $\mathcal{S}$-asymptotically $\omega$-periodic solutions of class $r$ for the nonlinear two-term time fractional differential equations with delay

$$
\begin{aligned}
& D_{t}^{\alpha+1} u(t)+\mu D_{t}^{\beta} u(t)-A u(t)=f\left(t, u_{t}\right), \quad t \in \mathbf{R}^{+}, 0<\alpha \leq \beta \leq 1, \mu>0 \\
& u_{0}=\varphi_{1} \in \mathscr{C}, \quad u_{0}^{\prime}=\varphi_{2} \in \mathscr{C}
\end{aligned}
$$

2010 Mathematics Subject Classification. 34A08, 35B40, 47D05.

Key words and phrases. Pseudo $\mathcal{S}$-asymptotically $\omega$-periodic solution; Fractional differential equations; Generalized semigroup theory; Sectorial operator.

This research is supported by the National Natural Science Foundation of China (Grant No. 11426201, 11271065) and the Natural Science Foundation of Zhejiang Province (Grant No. LQ13A010015).

Received September 16, 2014. 
where $A$ is an $\tilde{\omega}$-sectorial operator of angle $\beta \pi / 2$ with $\tilde{\omega}<0, u_{t}(\theta):=u(t+\theta)$ for $\theta \in[-r, 0], r \geq 0$ is a fixed constant, $\mathscr{C}:=C([-r, 0], \mathbf{R})$ denotes the space of continuous function from $[-r, 0]$ to $\mathbf{R}$ with the supremum norm. The fractional derivative is understood in the Caputo sense.

Note that fractional differential equations arise in many areas of applied science, such as physics, engineering, biology, control theory, among other areas. For this reason, those equations have been of a great interest during the last few decades. Our motivation to study (1.1) come from recent investigation on the subject. For (1.1) without delay, the existence, uniqueness of mild solutions in the special case $\alpha=\beta$ were studied in [15]; the nonlinear two-term time fractional diffusion-wave equation with $A=\frac{d^{2}}{d x^{2}}, 0<\alpha<\beta-1$ was studied in [20]; asymptotic behavior of mild solutions was studied in [14]; the $\mathcal{S}$-asymptotic $\omega$-periodicity of mild solutions was studied in [6]; the almost periodicity, compact almost automorphy, almost automorphy and pseudo asymptotic behavior of mild solutions were studied in [1], but in the case of delay, i.e., (1.1), to the best of our knowledge, there is no work reported in literature. Moreover, the pseudo $\mathcal{S}$ asymptotic $\omega$-periodicity of $(1.1)$ is quite new and an untreated topic. This is one of the key motivations of this study.

The paper is organized as follows. In Section 2, some notations and preliminary results are presented. In Section 3, we explore some properties of pseudo $\mathcal{S}$-asymptotically $\omega$-periodic function of class $r$, and establish the composition theorems. Section 4 is devoted to the existence, uniqueness of pseudo $\mathcal{S}$-asymptotically $\omega$-periodic mild solution of class $r$ for (1.1). In Section 5 , we present an application to a fractional partial differential equation with delay.

\section{Preliminaries and basic results}

Let $(X,\|\cdot\|),\left(Y,\|\cdot\|_{Y}\right)$ be two complex Banach spaces and $\mathbf{N}, \mathbf{R}, \mathbf{R}^{+}$, and $\mathbf{C}$ stand for the set of natural numbers, real numbers, nonnegative real numbers, and complex numbers, respectively. $\mathscr{R}(u)$ denotes the range of $u(\cdot)$. In order to facilitate the discussion below, we further introduce the following notations:

- $B C\left(\mathbf{R}^{+}, X\right)$ (resp. $\left.B C\left(\mathbf{R}^{+} \times Y, X\right)\right)$ : the Banach space of bounded continuous functions from $\mathbf{R}^{+}$to $X$ (resp. from $\mathbf{R}^{+} \times Y$ to $X$ ) with the supremum norm.

- $C\left(\mathbf{R}^{+}, X\right)$ (resp. $\left.C\left(\mathbf{R}^{+} \times Y, X\right)\right)$ : the set of continuous functions from $\mathbf{R}^{+}$ to $X$ (resp. from $\mathbf{R}^{+} \times Y$ to $X$ ).

- $L(X, Y)$ : the Banach space of bounded linear operators from $X$ to $Y$ endowed with the operator topology. In particular, we write $L(X)$ when $X=Y$.

- $L^{p}\left(\mathbf{R}^{+}, X\right)$ : the space of all classes of equivalence (with respect to the equality almost everywhere on $\mathbf{R}^{+}$) of measurable functions $f: \mathbf{R}^{+} \rightarrow X$ such that $\|f\| \in L^{p}\left(\mathbf{R}^{+}, \mathbf{R}^{+}\right)$. 
- $L_{l o c}^{p}\left(\mathbf{R}^{+}, X\right)$ : stand for the space of all classes of equivalence of measurable functions $f: \mathbf{R}^{+} \rightarrow X$ such that the restriction of $f$ to every bounded subinterval of $\mathbf{R}^{+}$is in $L^{p}\left(\mathbf{R}^{+}, X\right)$.

Let $p \in[1, \infty)$. The space $B S^{p}\left(\mathbf{R}^{+}, X\right)$ of all Stepanov bounded functions, with the exponent $p$, consists of all measurable functions $f: \mathbf{R}^{+} \rightarrow X$ such that $f^{b} \in L^{\infty}\left(\mathbf{R}^{+}, L^{p}([0,1] ; X)\right)$, where $f^{b}$ is the Bochner transform of $f$ defined by $f^{b}(t, s):=f(t+s), t \in \mathbf{R}^{+}, s \in[0,1] . \quad B S^{p}\left(\mathbf{R}^{+}, X\right)$ is a Banach space with the norm [17]

$$
\|f\|_{S^{p}}=\left\|f^{b}\right\|_{L^{\infty}\left(\mathbf{R}^{+}, L^{p}\right)}=\sup _{t \in \mathbf{R}^{+}}\left(\int_{t}^{t+1}\|f(\tau)\|^{p} \mathrm{~d} \tau\right)^{1 / p} .
$$

It is obvious that $L^{p}\left(\mathbf{R}^{+}, X\right) \subset B S^{p}\left(\mathbf{R}^{+}, X\right) \subset L_{l o c}^{p}\left(\mathbf{R}^{+}, X\right)$ and $B S^{p}\left(\mathbf{R}^{+}, X\right) \subset$ $B S^{q}\left(\mathbf{R}^{+}, X\right)$ for $p \geq q \geq 1$.

For each $p \in[1, \infty)$, we denote by $\mathscr{U} \mathscr{C}^{p}\left(\mathbf{R}^{+} \times Y, X\right)$ the set of all continuous functions $f: \mathbf{R}^{+} \times Y \rightarrow X$ with the property that there exists a function $L_{f} \in$ $B S^{p}\left(\mathbf{R}^{+}, \mathbf{R}^{+}\right)$satisfying $\forall \varepsilon>0, \exists \delta>0$ such that

$$
\begin{aligned}
& \sup _{\tau \in[t-r, t]}\|f(\tau, u(\tau))-f(\tau, v(\tau))\| \leq L_{f}(t) \varepsilon, \text { for all } t \in \mathbf{R}^{+} \text {and } \\
& u, v \in Y \text { with }\|u-v\|_{Y}<\delta .
\end{aligned}
$$

It is easy to see that $\mathscr{U} \mathscr{C}^{p}\left(\mathbf{R}^{+} \times Y, X\right) \subset \mathscr{U}_{\mathscr{C}} \mathscr{C}^{q}\left(\mathbf{R}^{+} \times Y, X\right)$ for $1 \leq q \leq p<\infty$.

Let $\alpha>0, m=\lceil\alpha\rceil$ denote the integer part of $\alpha$ and $u: \mathbf{R}^{+} \rightarrow X$. The Caputo fraction derivative of $u \in \mathbf{R}^{+}$of order $\alpha$ is defined by

$$
D_{t}^{\alpha} u(t)=\int_{0}^{t} g_{m-\alpha}(t-s) u^{(m)}(s) d s, \quad t>0,
$$

where $g_{\beta}(t):=\frac{t^{\beta-1}}{\Gamma(\beta)}, t>0, \beta>0, \Gamma$ is the Gamma function, and in case $\beta=0$, set $g_{0}(t):=\delta_{0}$, the Dirac measure concentrated at the origin.

In order to give an operator theoretical approach to (1.1), we have the following definition.

Definition 2.1 [14]. A closed and densely defined linear operator $A$ is said to $\tilde{\omega}$-sectorial of angle $\theta$ if there exists $\theta \in[0, \pi / 2)$ and $\tilde{\omega} \in \mathbf{R}$ such that its resolvent exists in the sector

$$
\begin{aligned}
& \tilde{\omega}+S_{\theta}:=\{\tilde{\omega}+\lambda: \lambda \in \mathbf{C},|\arg (\lambda)|<\pi / 2+\theta\} \backslash\{\tilde{\omega}\} \\
& \left\|(\lambda I-A)^{-1}\right\| \leq \frac{M}{|\lambda-\tilde{\omega}|}, \quad \lambda \in \tilde{\omega}+S_{\theta} .
\end{aligned}
$$

In the case $\tilde{\omega}=0$, we merely say that $A$ is sectorial of angle $\theta$. 
We should mention that in the general theory of sectorial operator, it is not require that (2.1) holds in a sector of angle $\pi / 2$. Our restriction corresponds to the class of operators used in this paper.

Definition 2.2 [14]. Let $\mu>0,0 \leq \alpha, \beta \leq 1$ be given. Let $A$ be a closed and linear operator with domain $D(A)$ defined on a Banach space $X$. We call $A$ the generator of an $(\alpha, \beta)_{\mu}$-regularized family if there exist $\tilde{\omega} \geq 0$ and a strongly continuous function $S_{\alpha, \beta}: \mathbf{R}^{+} \rightarrow L(X)$ such that $\left\{\lambda^{\alpha+1}+\mu \lambda^{\beta}: \operatorname{Re} \lambda>\tilde{\omega}\right\} \subset \rho(A)$ and

$$
\lambda^{\alpha}\left(\lambda^{\alpha+1}+\mu \lambda^{\beta}-A\right)^{-1} x=\int_{0}^{\infty} e^{-\lambda t} S_{\alpha, \beta}(t) x d t, \quad \operatorname{Re} \lambda>\tilde{\omega}, x \in X .
$$

Because of the uniqueness theorem for the Laplace transform, if $\mu=0$, $\alpha=0$, this corresponds to the case of a $C_{0}$-semigroup whereas the case $\mu=0$, $\alpha=1$ corresponds to the concept of cosine family. For more details on the Laplace transform approach to semigroups and cosine functions, we refer to [2].

Sufficient conditions to existence and the integrability for the generators of an $(\alpha, \beta)_{\mu}$-regularized family are given in the following results.

Theorem 2.1 [14]. Let $0<\alpha \leq \beta \leq 1, \mu>0$ and $\tilde{\omega}<0$. Assume that $A$ is an $\tilde{\omega}$-sectorial of angle $\beta \pi / 2$, then $A$ generates an $(\alpha, \beta)_{\mu}$-regularized family $S_{\alpha, \beta}(t)$ satisfying

$$
\left\|S_{\alpha, \beta}(t)\right\| \leq \frac{C}{1+|\tilde{\omega}|\left(t^{\alpha+1}+\mu t^{\beta}\right)}, \quad t \geq 0
$$

for some constant $C>0$ depending only on $\alpha, \beta$.

Note that

$$
\int_{0}^{\infty} \frac{1}{1+|\tilde{\omega}| t^{\alpha+1}} d t=\frac{|\tilde{\omega}|^{-1 /(\alpha+1)} \pi}{(\alpha+1) \sin (\pi /(\alpha+1))}
$$

for $0<\alpha<1$, therefore $S_{\alpha, \beta}(t)$ is integrable on $(0, \infty)$.

THEOREM 2.2 (Hardy-Littlewood [2]). Let $f \in L_{l o c}^{1}\left(\mathbf{R}^{+}, X\right)$ and $F(t):=$ $\int_{0}^{t} f(s) d s$. Assume that $M:=\sup _{t \geq \tau} t\|f(t)\|<\infty$ for some $\tau \geq 0$ and $F_{\infty} \in X$. If $\lim _{\lambda \rightarrow 0} \hat{f}(\lambda)=F_{\infty}$, then $\lim _{t \rightarrow \infty} F(t)=F_{\infty}$, where $\hat{f}(\lambda):=\int_{0}^{\infty} e^{-\lambda t} f(t) d t$.

\section{Pseudo $\mathscr{S}$-asymptotic $\omega$-periodicity of class $r$}

For $\omega>0$, define

$$
\begin{aligned}
& C_{0}\left(\mathbf{R}^{+}, X\right)=\left\{f \in B C\left(\mathbf{R}^{+}, X\right): \lim _{t \rightarrow \infty}\|f(t)\|=0\right\} . \\
& C_{\omega}\left(\mathbf{R}^{+}, X\right)=\left\{f \in B C\left(\mathbf{R}^{+}, X\right): f \text { is } \omega \text {-periodic }\right\} .
\end{aligned}
$$


Definition 3.1 [21]. A function $f \in B C\left(\mathbf{R}^{+}, X\right)$ is called asymptotically $\omega$ periodic if there exist $g \in C_{\omega}\left(\mathbf{R}^{+}, X\right), \varphi \in C_{0}\left(\mathbf{R}^{+}, X\right)$ such that $f=g+\varphi$. The collection of those functions is denoted by $A P_{\omega}\left(\mathbf{R}^{+}, X\right)$.

Definition 3.2 [10]. A function $f \in B C\left(\mathbf{R}^{+}, X\right)$ is said to be $\mathcal{S}$ asymptotically periodic if there exists $\omega>0$ such that $\lim _{t \rightarrow \infty}(f(t+\omega)-f(t))$ $=0$. In this case, we say that $f$ is $\mathcal{S}$-asymptotically $\omega$-periodic. The collection of those functions is denoted by $\mathcal{S} A P_{\omega}\left(\mathbf{R}^{+}, X\right)$. It is clear that $A P_{\omega}\left(\mathbf{R}^{+}, X\right) \subset$ $\mathcal{S} A P_{\omega}\left(\mathbf{R}^{+}, X\right)$.

Definition 3.3 [19]. A function $f \in B C\left(\mathbf{R}^{+}, X\right)$ is called pseudo $\mathcal{S}$ asymptotically periodic if there exists $\omega>0$ such that

$$
\lim _{T \rightarrow \infty} \frac{1}{T} \int_{0}^{T}\|f(t+\omega)-f(t)\| d t=0 .
$$

In this case, we say that $f$ is pseudo $\mathcal{S}$-asymptotically $\omega$-periodic. Denote by $P \mathcal{S} A P_{\omega}\left(\mathbf{R}^{+}, X\right)$ the set of such functions, $P \mathcal{S} A P_{\omega}\left(\mathbf{R}^{+}, X\right)$ is a Banach space when endowed with the supremum norm and $\mathcal{S} A P_{\omega}\left(\mathbf{R}^{+}, X\right) \subset P \mathcal{S} A P_{\omega}\left(\mathbf{R}^{+}, X\right)$.

Definition 3.4 [19]. A function $f \in B C\left(\mathbf{R}^{+}, X\right)$ is called pseudo $\mathcal{S}$ asymptotically $\omega$-periodic of class $r(r \geq 0)$ if there exists $\omega>0$ such that

$$
\lim _{T \rightarrow \infty} \frac{1}{T} \int_{r}^{T} \sup _{\tau \in[t-r, t]}\|f(\tau+\omega)-f(\tau)\| d t=0 .
$$

Denote by $P \mathcal{S} A P_{\omega, r}\left(\mathbf{R}^{+}, X\right)$ the set of such functions, $P \mathcal{S} A P_{\omega, r}\left(\mathbf{R}^{+}, X\right)$ is a Banach space when endowed with the supremum norm.

Definition 3.5 [19]. A function $F \in B C\left(\mathbf{R}^{+} \times Y, X\right)$ is called uniformly pseudo $\mathcal{S}$-asymptotically $\omega$-periodic of class $r(r \geq 0)$ if there exists $\omega>0$ such that

$$
\lim _{T \rightarrow \infty} \frac{1}{T} \int_{r}^{T} \sup _{\tau \in[t-r, t]} \sup _{\|z\|_{Y} \leq R}\|F(\tau+\omega, z)-F(\tau, z)\| d t=0,
$$

for all $R>0$. Denote by $P \mathcal{S} A P_{\omega, r}\left(\mathbf{R}^{+} \times Y, X\right)$ the set of such functions.

Next, we show some properties of the space $P \mathcal{S} A P_{\omega, r}\left(\mathbf{R}^{+}, X\right)$.

LEMMA 3.1. Let $r \geq 0$, then

(i) $P \mathcal{S} A P_{\omega, r}\left(\mathbf{R}^{+}, X\right) \subseteq P \mathcal{S A P} P_{\omega}\left(\mathbf{R}^{+}, X\right)$.

(ii) $P \mathcal{S} A P_{\omega, r}\left(\mathbf{R}^{+}, X\right)$ is a closed subspace of $B C\left(\mathbf{R}^{+}, X\right)$.

(iii) $P \mathcal{S} A P_{\omega, r}\left(\mathbf{R}^{+}, X\right)$ is a Banach space under the supermum norm.

Proof. From the estimate 


$$
\begin{aligned}
& \frac{1}{T} \int_{0}^{T}\|f(t+\omega)-f(t)\| d t \\
& \quad=\frac{1}{T} \int_{0}^{r}\|f(t+\omega)-f(t)\| d t+\frac{1}{T} \int_{r}^{T}\|f(t+\omega)-f(t)\| d t \\
& \quad \leq \frac{1}{T} \int_{0}^{r}\|f(t+\omega)-f(t)\| d t+\frac{1}{T} \int_{r}^{T} \sup _{\tau \in[t-r, t]}\|f(\tau+\omega)-f(\tau)\| d t
\end{aligned}
$$

it is easy to see that (i) holds.

Let $f_{n} \in P \mathcal{S} A P_{\omega, r}\left(\mathbf{R}^{+}, X\right)$ and $f_{n} \rightarrow f$ in $B C\left(\mathbf{R}^{+}, X\right)$, then

$$
\begin{aligned}
\frac{1}{T} \int_{r}^{T} & \sup _{\tau \in[t-r, t]}\|f(\tau+\omega)-f(\tau)\| d t \\
= & \frac{1}{T} \int_{r}^{T} \sup _{\tau \in[t-r, t]}\left\|f_{n}(\tau+\omega)-f_{n}(\tau)\right\| d t \\
& +\frac{1}{T} \int_{r}^{T} \sup _{\tau \in[t-r, t]}\left\|f(\tau+\omega)-f(\tau)-\left(f_{n}(\tau+\omega)-f_{n}(\tau)\right)\right\| d t \\
\leq & \frac{1}{T} \int_{r}^{T} \sup _{\tau \in[t-r, t]}\left\|f_{n}(\tau+\omega)-f_{n}(\tau)\right\| d t \\
& +\frac{1}{T} \int_{r}^{T} \sup _{\tau \in[t-r, t]}\left\|f(\tau+\omega)-f_{n}(\tau+\omega)\right\| d t \\
& +\frac{1}{T} \int_{r}^{T} \sup _{\tau \in[t-r, t]}\left\|f(\tau)-f_{n}(\tau)\right\| d t,
\end{aligned}
$$

which yields that $f \in P \mathcal{S} A P_{\omega, r}\left(\mathbf{R}^{+}, X\right)$, then (ii) holds, therefore (iii) holds.

LEMMA 3.2. Let $r_{1}>0, r_{2}>0$, then

$$
P \mathcal{S} A P_{\omega, r_{1}}\left(\mathbf{R}^{+}, X\right)=P \mathcal{S} A P_{\omega, r_{2}}\left(\mathbf{R}^{+}, X\right) .
$$

Proof. Let $r>0$, first we show that

$$
P \mathcal{S} A P_{\omega, r}\left(\mathbf{R}^{+}, X\right) \subset P \mathcal{S} A P_{\omega, 2 r}\left(\mathbf{R}^{+}, X\right) .
$$

For $f \in P \mathcal{S} A P_{\omega, r}\left(\mathbf{R}^{+}, X\right)$, one has

$$
\begin{aligned}
& \frac{1}{T} \int_{2 r}^{T} \sup _{\tau \in[t-2 r, t]}\|f(\tau+\omega)-f(\tau)\| d t \\
& \quad \leq \frac{1}{T} \int_{2 r}^{T} \sup _{\tau \in[t-r, t]}\|f(\tau+\omega)-f(\tau)\| d t+\frac{1}{T} \int_{2 r}^{T} \sup _{\tau \in[t-2 r, t-r]}\|f(\tau+\omega)-f(\tau)\| d t
\end{aligned}
$$




$$
\begin{aligned}
& \leq \frac{1}{T} \int_{r}^{T} \sup _{\tau \in[t-r, t]}\|f(\tau+\omega)-f(\tau)\| d t+\frac{1}{T} \int_{r}^{T-r} \sup _{\tau \in[u-r, u]}\|f(\tau+\omega)-f(\tau)\| d u \\
& \leq \frac{1}{T} \int_{r}^{T} \sup _{\tau \in[t-r, t]}\|f(\tau+\omega)-f(\tau)\| d t+\frac{1}{T} \int_{r}^{T} \sup _{\tau \in[u-r, u]}\|f(\tau+\omega)-f(\tau)\| d u,
\end{aligned}
$$

so

$$
\lim _{T \rightarrow \infty} \frac{1}{T} \int_{2 r}^{T} \sup _{\tau \in[t-2 r, t]}\|f(\tau+\omega)-f(\tau)\| d t=0,
$$

thus $f \in P \mathcal{S} A P_{\omega, 2 r}\left(\mathbf{R}^{+}, X\right)$. Hence (3.1) holds.

Now, let $r_{1}>r_{2}>0$. If $f \in P \mathcal{S} A P_{\omega, r_{1}}\left(\mathbf{R}^{+}, X\right)$, then

$$
\lim _{T \rightarrow \infty} \frac{1}{T} \int_{r_{1}}^{T} \sup _{\tau \in\left[t-r_{1}, t\right]}\|f(\tau+\omega)-f(\tau)\| d t=0 .
$$

From

$$
\begin{aligned}
& \frac{1}{T} \int_{r_{2}}^{T} \sup _{\tau \in\left[t-r_{2}, t\right]}\|f(\tau+\omega)-f(\tau)\| d t \\
& \quad=\frac{1}{T} \int_{r_{2}}^{r_{1}} \sup _{\tau \in\left[t-r_{2}, t\right]}\|f(\tau+\omega)-f(\tau)\| d t+\frac{1}{T} \int_{r_{1}}^{T} \sup _{\tau \in\left[t-r_{2}, t\right]}\|f(\tau+\omega)-f(\tau)\| d t \\
& \quad \leq \frac{1}{T} \int_{r_{2}}^{r_{1}} \sup _{\tau \in\left[t-r_{2}, t\right]}\|f(\tau+\omega)-f(\tau)\| d t+\frac{1}{T} \int_{r_{1}}^{T} \sup _{\tau \in\left[t-r_{1}, t\right]}\|f(\tau+\omega)-f(\tau)\| d t
\end{aligned}
$$

then

$$
\lim _{T \rightarrow \infty} \frac{1}{T} \int_{r_{2}}^{T} \sup _{\tau \in\left[t-r_{2}, t\right]}\|f(\tau+\omega)-f(\tau)\| d t=0,
$$

so $f \in P \mathcal{S} A P_{\omega, r_{2}}\left(\mathbf{R}^{+}, X\right)$, i.e., one has

$$
P \mathcal{S} A P_{\omega, r_{1}}\left(\mathbf{R}^{+}, X\right) \subset P \mathcal{S} A P_{\omega, r_{2}}\left(\mathbf{R}^{+}, X\right) .
$$

On the other hand, since $r_{1}>r_{2}$, there exists $k \in \mathbf{N}$ such that $2^{k} r_{2}>r_{1}$. By (3.1), (3.2), one has

$$
P \mathcal{S} A P_{\omega, r_{2}}\left(\mathbf{R}^{+}, X\right) \subset P \mathcal{S} A P_{\omega, 2^{k} r_{2}}\left(\mathbf{R}^{+}, X\right) \subset P \mathcal{S} A P_{\omega, r_{1}}\left(\mathbf{R}^{+}, X\right) .
$$

Thus, $P \mathcal{S} A P_{\omega, r_{1}}\left(\mathbf{R}^{+}, X\right)=P \mathcal{S} A P_{\omega, r_{2}}\left(\mathbf{R}^{+}, X\right)$. The proof is complete.

Remark 3.1. It is interesting that $P \mathcal{S} A P_{\omega, r}\left(\mathbf{R}^{+}, X\right)=P \mathcal{S} A P_{\omega, 1}\left(\mathbf{R}^{+}, X\right)$ for all $r>0$ by Lemma 3.2, but for $r=0$, it is not necessarily holds, i.e., $P \mathcal{S} A P_{\omega, 0}\left(\mathbf{R}^{+}, X\right)=P \mathcal{S} A P_{\omega, 1}\left(\mathbf{R}^{+}, X\right)$ is not true. 
LEMMA 3.3. Let $f \in P \mathcal{S} A P_{\omega, r}\left(\mathbf{R}^{+}, X\right)$, then $f(\cdot+\eta) \in P \mathcal{S} A P_{\omega, r}\left(\mathbf{R}^{+}, X\right)$ for all $\eta \geq 0$.

Proof. For $\eta \geq 0$,

$$
\begin{aligned}
\frac{1}{T} \int_{r}^{T} & \sup _{\tau \in[t-r, t]}\|f(\tau+\eta+\omega)-f(\tau+\eta)\| d t \\
= & \frac{1}{T} \int_{r}^{T} \sup _{\tau \in[t+\eta-r, t+\eta]}\|f(\tau+\omega)-f(\tau)\| d t \\
= & \frac{1}{T} \int_{r+\eta}^{T+\eta} \sup _{\tau \in[t-r, t]}\|f(\tau+\omega)-f(\tau)\| d t \\
= & \frac{T+\eta}{T} \cdot \frac{1}{T+\eta} \int_{r}^{T+\eta} \sup _{\tau \in[t-r, t]}\|f(\tau+\omega)-f(\tau)\| d t \\
& -\frac{1}{T} \int_{r}^{r+\eta} \sup _{\tau \in[t-r, t]}\|f(\tau+\omega)-f(\tau)\| d t,
\end{aligned}
$$

then

$$
\lim _{T \rightarrow \infty} \frac{1}{T} \int_{r}^{T} \sup _{\tau \in[t-r, t]}\|f(\tau+\eta+\omega)-f(\tau+\eta)\| d t=0,
$$

which implies that $f(\cdot+\eta) \in P \mathcal{S} A P_{\omega, r}\left(\mathbf{R}^{+}, X\right)$ for all $\eta \geq 0$.

We will establish some composition theorems for pseudo $\mathcal{S}$-asymptotically $\omega$ periodic function of class $r$.

THEOREM 3.1. Let $f \in P \mathcal{S} A P_{\omega, r}\left(\mathbf{R}^{+} \times Y, X\right)$ and there exists a constant $L_{f}>0$ such that

$$
\|f(t, u)-f(t, v)\| \leq L_{f}\|u-v\|_{Y}, \quad t \in \mathbf{R}^{+}, u, v \in Y,
$$

then $h(\cdot)=f(\cdot, u(\cdot)) \in P \mathcal{S} A P_{\omega, r}\left(\mathbf{R}^{+}, X\right)$ if $u(\cdot) \in P \mathcal{S A P} P_{\omega, r}\left(\mathbf{R}^{+}, Y\right)$.

Proof. It is clear that $f(\cdot, u(\cdot)) \in B C\left(\mathbf{R}^{+}, X\right)$. For $\varepsilon>0$, there exists $L_{\varepsilon}>0$ such that

$$
\begin{aligned}
& \frac{1}{T} \int_{r}^{T} \sup _{\tau \in[t-r, t]} \sup _{\|z\| \leq R}\|f(\tau+\omega, z)-f(\tau, z)\| d t \leq \varepsilon, \\
& \frac{1}{T} \int_{r}^{T} \sup _{\tau \in[t-r, t]}\|u(\tau+\omega)-u(\tau)\|_{Y} d t \leq \varepsilon / L_{f},
\end{aligned}
$$

for every $T \geq L_{\varepsilon}, R>0$. For $T \geq L_{\varepsilon}$, 


$$
\begin{aligned}
& \frac{1}{T} \int_{r}^{T} \sup _{\tau \in[t-r, t]}\|f(\tau+\omega, u(\tau+\omega))-f(\tau, u(\tau))\| d t \\
& \leq \frac{1}{T} \int_{r}^{T} \sup _{\tau \in[t-r, t]}\|f(\tau+\omega, u(\tau+\omega))-f(\tau, u(\tau+\omega))\| d t \\
& \quad+\frac{1}{T} \int_{r}^{T} \sup _{\tau \in[t-r, t]}\|f(\tau, u(\tau+\omega))-f(\tau, u(\tau))\| d t \\
& \leq \frac{1}{T} \int_{r}^{T} \sup _{\tau \in[t-r, t]} \sup _{\|z\| \leq R}\|f(\tau+\omega, z)-f(\tau, z)\| d t \\
& \quad+\frac{L_{f}}{T} \int_{r}^{T} \sup _{\tau \in[t-r, t]}\|u(\tau+\omega)-u(\tau)\|_{Y} d t \\
& \leq 2 \varepsilon,
\end{aligned}
$$

implies that $h(\cdot) \in P \mathcal{S} A P_{\omega, r}\left(\mathbf{R}^{+}, X\right)$.

In the following, we establish another composition theorem which weakens the assumptions on $f$.

Lemma 3.4. Let $f \in B C\left(\mathbf{R}^{+}, X\right)$, then $f \in P \mathcal{S} A P_{\omega, r}\left(\mathbf{R}^{+}, X\right)$ if and only if for any $\varepsilon>0$,

$$
\lim _{T \rightarrow \infty} \frac{1}{T} \operatorname{mes}\left(M_{T, \varepsilon}(f)\right)=0,
$$

where $\operatorname{mes}(\cdot)$ denotes the Lebesgue measure and

$$
M_{T, \varepsilon}(f)=\left\{t \in[r, T]: \sup _{\tau \in[t-r, t]}\|f(\tau+\omega)-f(\tau)\| \geq \varepsilon\right\} .
$$

Proof. Sufficiency: From the statement of the Lemma it is clear that $\|f\|<\infty$ and for any $\varepsilon>0$, there exists $T_{0}>0$ such that for $T>T_{0}$,

$$
\frac{1}{T} \operatorname{mes}\left(M_{T, \varepsilon}(f)\right)<\frac{\varepsilon}{2\|f\|} .
$$

Then,

$$
\begin{aligned}
& \frac{1}{T} \int_{r}^{T} \sup _{\tau \in[t-r, t]}\|f(\tau+\omega)-f(\tau)\| d t \\
&=\frac{1}{T}\left(\int_{M_{T, \varepsilon}} \sup _{\tau \in[t-r, t]}\|f(\tau+\omega)-f(\tau)\| d t\right. \\
&\left.\quad+\int_{[r, T] \backslash M_{T, \varepsilon}} \sup _{\tau \in[t-r, t]}\|f(\tau+\omega)-f(\tau)\| d t\right)
\end{aligned}
$$




$$
\begin{aligned}
& \leq \frac{2\|f\|}{T} \operatorname{mes}\left(M_{T, \varepsilon}(f)\right)+\frac{\varepsilon}{T} \int_{[r, T] \backslash M_{T, \varepsilon}} d t \\
& \leq \frac{2\|f\|}{T} \operatorname{mes}\left(M_{T, \varepsilon}(f)\right)+\frac{\varepsilon}{T} \int_{0}^{T} d t \\
& \leq 2 \varepsilon
\end{aligned}
$$

so

$$
\lim _{T \rightarrow \infty} \frac{1}{T} \int_{r}^{T} \sup _{\tau \in[t-r, t]}\|f(\tau+\omega)-f(\tau)\| d t=0 .
$$

That is $f \in P \mathcal{S} A P_{\omega, r}\left(\mathbf{R}^{+}, X\right)$.

1 Necessity: Suppose the contrary, that there exists $\varepsilon_{0}>0$, such that $\frac{1}{T} \operatorname{mes}\left(M_{T, \varepsilon}(f)\right)$ does not converge to 0 as $T \rightarrow \infty$. That is there exists $\delta>0$, such that for each $n$,

$$
\frac{1}{T_{n}} \operatorname{mes}\left(M_{T_{n}, \varepsilon_{0}}(f)\right) \geq \delta \text { for some } T_{n}>n,
$$

then

$$
\begin{aligned}
\frac{1}{T_{n}} \int_{r}^{T_{n}} \sup _{\tau \in[t-r, t]}\|f(\tau+\omega)-f(\tau)\| d t \\
\quad=\frac{1}{T_{n}} \int_{M_{T_{n}, \varepsilon_{0}}} \sup _{\tau \in[t-r, t]}\|f(\tau+\omega)-f(\tau)\| d t \\
\quad+\int_{\left[r, T_{n}\right] \backslash M_{T_{n}, \varepsilon_{0}}} \sup _{\tau \in[t-r, t]}\|f(\tau+\omega)-f(\tau)\| d t \\
\geq \frac{1}{T_{n}} \int_{M_{T_{n}, \varepsilon_{0}}} \sup _{\tau \in[t-r, t]}\|f(\tau+\omega)-f(\tau)\| d t \\
\geq \frac{1}{T_{n}} \operatorname{mes}\left(M_{T_{n}, \varepsilon_{0}}(f)\right) \varepsilon_{0} \\
\geq \varepsilon_{0} \delta,
\end{aligned}
$$

which contradicts the fact that

$$
\lim _{T \rightarrow \infty} \frac{1}{T} \int_{r}^{T} \sup _{\tau \in[t-r, t]}\|f(\tau+\omega)-f(\tau)\| d t=0 .
$$

Thus (3.3) holds.

THEOREM 3.2. Assume that $f \in P \mathcal{S} A P_{\omega, r}\left(\mathbf{R}^{+} \times Y, X\right) \cap \mathscr{U}_{\mathscr{C}}{ }^{p}\left(\mathbf{R}^{+} \times Y, X\right)$, $p \in[1, \infty)$, then $h(\cdot)=f(\cdot, u(\cdot)) \in P \mathcal{S} A P_{\omega, r}\left(\mathbf{R}^{+}, X\right)$ if $u \in P \mathcal{S} A P_{\omega, r}\left(\mathbf{R}^{+}, Y\right)$. 
Proof. It is clear that $f(\cdot, u(\cdot)) \in B C\left(\mathbf{R}^{+}, X\right)$. Moreover, since $f \in$ $P \mathcal{S} A P_{\omega, r}\left(\mathbf{R}^{+} \times Y, X\right) \cap \mathscr{U} \mathscr{C}^{p}\left(\mathbf{R}^{+} \times Y, X\right)$, for $\forall \varepsilon>0$, there exists $L_{\varepsilon}>0, \delta>0$, $L_{f} \in B S^{p}\left(\mathbf{R}^{+}, \mathbf{R}^{+}\right)$such that

$$
\begin{aligned}
& \frac{1}{T} \int_{r}^{T} \sup _{\tau \in[t-r, t]} \sup _{\|z\|_{Y} \leq R}\|f(\tau+\omega, z)-f(\tau, z)\| d t \leq \varepsilon, \quad \text { for every } T \geq L_{\varepsilon}, R>0 . \\
& \|F(t)\|:=\sup _{\tau \in[t-r, t]}\|f(\tau, u(\tau+\omega))-f(\tau, u(\tau))\| \leq L_{f}(t) \varepsilon, \quad \text { if } \\
& \|z(t)\|_{Y}:=\|u(t+\omega)-u(t)\|_{Y}<\delta, \quad t \in \mathbf{R}^{+} .
\end{aligned}
$$

Denote

$$
\begin{aligned}
& M_{T, \delta}(z)=\left\{t \in[r, T]: \sup _{\tau \in[t-r, t]}\|u(\tau+\omega)-u(\tau)\|_{Y} \geq \delta\right\} . \\
& \tilde{M}_{T, \delta}(z)=\left\{t \in[r, T]:\|u(t+\omega)-u(t)\|_{Y} \geq \delta\right\} .
\end{aligned}
$$

then $\tilde{M}_{T, \delta}(z) \subset M_{T, \delta}(z)$ and $\lim _{T \rightarrow \infty} \frac{1}{T} \operatorname{mes}\left(M_{T, \delta}(z)\right)=0$ by Lemma 3.4 .
So

$$
\begin{aligned}
& \frac{1}{T} \int_{r}^{T} \sup _{\tau \in[t-r, t]}\|f(\tau, u(\tau+\omega))-f(\tau, u(\tau))\| d t \\
& \leq \frac{1}{T} \int_{\tilde{M}_{T, \delta}(z)} \sup _{\tau \in[t-r, t]}\|f(\tau, u(\tau+\omega))-f(\tau, u(\tau))\| d t \\
&+\frac{1}{T} \int_{[r, T] \backslash \tilde{M}_{T, \delta}(z)} \sup _{\tau \in[t-r, t]}\|f(\tau, u(\tau+\omega))-f(\tau, u(\tau))\| d t \\
& \leq \frac{\|F\|_{\infty}}{T} \operatorname{mes}\left(\tilde{M}_{T, \delta}(z)\right)+\frac{\varepsilon}{T} \int_{r}^{T} L_{f}(t) d t \\
& \leq \frac{\|F\|_{\infty}}{T} \operatorname{mes}\left(M_{T, \delta}(z)\right)+\frac{\varepsilon}{T} \sum_{k=0}^{[T]} \int_{k}^{k+1} L_{f}(t) d t \\
& \leq \frac{\|F\|_{\infty}}{T} \operatorname{mes}\left(M_{T, \delta}(z)\right)+\frac{\varepsilon}{T} \sum_{k=0}^{[T]}\left(\int_{k}^{k+1} L_{f}(t)^{p} d t\right)^{1 / p} \\
& \leq \frac{\|F\|_{\infty}}{T} \operatorname{mes}\left(M_{T, \delta}(z)\right)+\frac{[T]+1}{T}\left\|L_{f}\right\|_{S^{p}} \varepsilon,
\end{aligned}
$$

where $\|F\|_{\infty}:=\sup _{t \in \mathbf{R}^{+}}\|F(t)\|$.

For $T \geq L_{\varepsilon}$, by (3.4), one has 
FRACTIONAL DIFFERENTIAL EQUATIONS WITH DELAY

$$
\begin{aligned}
\frac{1}{T} \int_{r}^{T} & \sup _{\tau \in[t-r, t]}\|f(\tau+\omega, u(\tau+\omega))-f(\tau, u(\tau))\| d t \\
\leq & \frac{1}{T} \int_{r}^{T} \sup _{\tau \in[t-r, t]}\|f(\tau+\omega, u(\tau+\omega))-f(\tau, u(\tau+\omega))\| d t \\
& +\frac{1}{T} \int_{r}^{T} \sup _{\tau \in[t-r, t]}\|f(\tau, u(\tau+\omega))-f(\tau, u(\tau))\| d t \\
\leq & +\frac{\|F\|_{\infty}}{T} \operatorname{mes}\left(M_{T, \delta}(z)\right)+\frac{[T]+1}{T}\left\|L_{f}\right\|_{S^{p}} \varepsilon,
\end{aligned}
$$

Due to the arbitrariness of $\varepsilon$, one has

$$
\lim _{T \rightarrow \infty} \frac{1}{T} \int_{r}^{T} \sup _{\tau \in[t-r, t]}\|f(\tau+\omega, u(\tau+\omega))-f(\tau, u(\tau))\| d t=0,
$$

implies that $h(\cdot) \in P \mathcal{S} A P_{\omega, r}\left(\mathbf{R}^{+}, X\right)$.

LEMMA 3.5. Let $u \in B C([-r, \infty), X)$ and assume that $\left.u\right|_{[0, \infty)} \in$ $P \mathcal{S} A P_{\omega, r}\left(\mathbf{R}^{+}, X\right)$, then $u_{t} \in P \mathcal{S} A P_{\omega, r}\left(\mathbf{R}^{+}, \mathscr{C}\right)$.

Proof. Note that

$$
\begin{aligned}
\frac{1}{T} \int_{r}^{T} & \sup _{\tau \in[t-r, t]}\left\|u_{\tau+\omega}-u_{\tau}\right\|_{\mathscr{C}} d t \\
\leq & \frac{1}{T} \int_{r}^{T} \sup _{\tau \in[t-r, t]}\left(\sup _{\theta \in[-r, 0]}\|u(\tau+\omega+\theta)-u(\tau+\theta)\|\right) d t \\
\leq & \frac{1}{T} \int_{r}^{T} \sup _{\tau \in[t-2 r, t]}\|u(\tau+\omega)-u(\tau)\| d t \\
\leq & \frac{1}{T} \int_{r}^{T} \sup _{\tau \in[t-2 r, t-r]}\|u(\tau+\omega)-u(\tau)\| d t+\frac{1}{T} \int_{r}^{T} \sup _{\tau \in[t-r, t]}\|u(\tau+\omega)-u(\tau)\| d t \\
= & \frac{1}{T} \int_{0}^{T-r} \sup _{\tau \in[t-r, t]}\|u(\tau+\omega)-u(\tau)\| d t+\frac{1}{T} \int_{r}^{T} \sup _{\tau \in[t-r, t]}\|u(\tau+\omega)-u(\tau)\| d t \\
= & \frac{1}{T} \int_{0}^{r} \sup _{\tau \in[t-r, t]}\|u(\tau+\omega)-u(\tau)\| d t+\frac{1}{T} \int_{r}^{T-r} \sup _{\tau \in[t-r, t]}\|u(\tau+\omega)-u(\tau)\| d t \\
& +\frac{1}{T} \int_{r}^{T} \sup _{\tau \in[t-r, t]}\|u(\tau+\omega)-u(\tau)\| d t \\
\leq & \frac{1}{T} \int_{0}^{r} \sup _{\tau \in[t-r, t]}\|u(\tau+\omega)-u(\tau)\| d t+\frac{2}{T} \int_{r}^{T} \sup _{\tau \in[t-r, t]}\|u(\tau+\omega)-u(\tau)\| d t,
\end{aligned}
$$


By $\left.u\right|_{[0, \infty)} \in P \mathcal{S} A P_{\omega, r}\left(\mathbf{R}^{+}, X\right)$, it is easy to see that

$$
\lim _{T \rightarrow \infty} \frac{1}{T} \int_{r}^{T} \sup _{\tau \in[t-r, t]}\left\|u_{\tau+\omega}-u_{\tau}\right\|_{\mathscr{C}} d t=0 .
$$

Hence $u_{t} \in P \mathcal{S} A P_{\omega, r}\left(\mathbf{R}^{+}, \mathscr{C}\right)$. The proof is complete.

Lemma 3.6. Let $\{S(t)\}_{t \geq 0} \subset L(X)$ be a strongly continuous family of bounded and linear operators such that $\|S(t)\| \leq \phi(t), t \in \mathbf{R}^{+}$, where $\phi \in L^{1}\left(\mathbf{R}^{+}\right)$ is nonincreasing. If $f \in P \mathcal{S A P} P_{\omega, r}\left(\mathbf{R}^{+}, X\right)$, then

$$
(\Lambda f)(t):=\int_{0}^{t} S(t-s) f(s) d s \in P \mathcal{S} A P_{\omega, r}\left(\mathbf{R}^{+}, X\right), \quad t \in \mathbf{R}^{+} .
$$

Proof. Note that

$$
\begin{aligned}
(\Lambda f)(\tau+\omega)-(\Lambda f)(\tau) & =\int_{0}^{\omega} S(\tau+\omega-s) f(s) d s+\int_{0}^{\tau} S(\tau-s)[f(s+\omega)-f(s)] d s \\
& :=I(\tau)+J(\tau),
\end{aligned}
$$

where

$$
I(\tau)=\int_{0}^{\omega} S(\tau+\omega-s) f(s) d s, \quad J(\tau)=\int_{0}^{\tau} S(\tau-s)[f(s+\omega)-f(s)] d s .
$$

Since

$$
\begin{aligned}
\frac{1}{T} \int_{r}^{T} \sup _{\tau \in[t-r, t]}\|I(\tau)\| d t & \leq \frac{1}{T} \int_{r}^{T} \sup _{\tau \in[t-r, t]}\left(\int_{0}^{\omega} \phi(\tau+\omega-s)\|f(s)\| d s\right) d t \\
& \leq \frac{1}{T} \int_{r}^{T} \sup _{\tau \in[t-r, t]} \phi(\tau)\left(\int_{0}^{\omega}\|f(s)\| d s\right) d t \\
& \leq \frac{1}{T} \int_{r}^{T} \phi(t-r)\left(\int_{0}^{\omega}\|f(s)\| d s\right) d t \\
& =\frac{1}{T} \int_{0}^{T-r} \phi(t) d t \cdot\left(\int_{0}^{\omega}\|f(s)\| d s\right) \\
& \leq \frac{\|\phi\|_{L^{1}}}{T}\left(\int_{0}^{\omega}\|f(s)\| d s\right) \rightarrow 0, \quad T \rightarrow \infty,
\end{aligned}
$$

then

$$
\lim _{T \rightarrow \infty} \frac{1}{T} \int_{r}^{T} \sup _{\tau \in[t-r, t]}\|I(\tau)\| d t=0 .
$$


Next, we will prove that

$$
\lim _{T \rightarrow \infty} \frac{1}{T} \int_{r}^{T} \sup _{\tau \in[t-r, t]}\|J(\tau)\| d t=0 .
$$

Since $f \in P \mathcal{S} A P_{\omega, r}\left(\mathbf{R}^{+}, X\right), f \in P \mathcal{S} A P_{\omega}\left(\mathbf{R}^{+}, X\right)$ by Lemma 3.1, then

$$
\begin{aligned}
& \lim _{T \rightarrow \infty} \frac{1}{T} \int_{0}^{T}\|f(t+\omega)-f(t)\| d t=0 . \\
& \lim _{T \rightarrow \infty} \frac{1}{T} \int_{r}^{T} \sup _{\tau \in[t-r, t]}\|f(\tau+\omega)-f(\tau)\| d t=0 .
\end{aligned}
$$

Define

$$
\varphi_{T}(s):=\frac{1}{T} \int_{0}^{T-s}\|f(t+\omega)-f(t)\| d t, \quad s \in \mathbf{R}^{+},
$$

then $\varphi_{T}(s)$ is decreasing on $\mathbf{R}^{+}$and

$$
\lim _{T \rightarrow \infty} \varphi_{T}(0)=\lim _{T \rightarrow \infty} \frac{1}{T} \int_{0}^{T}\|f(t+\omega)-f(t)\| d t=0 .
$$

Moreover,

$$
\begin{aligned}
\frac{1}{T} \int_{r}^{T} \sup _{\tau \in[t-r, t]}\|J(\tau)\| d t \leq & \frac{1}{T} \int_{r}^{T} \sup _{\tau \in[t-r, t]}\left(\int_{0}^{\tau}\|S(\tau-s)\|\|f(s+\omega)-f(s)\| d s\right) d t \\
\leq & \frac{1}{T} \int_{r}^{T} \sup _{\tau \in[t-r, t]}\left(\int_{0}^{\tau} \phi(\tau-s)\|f(s+\omega)-f(s)\| d s\right) d t \\
= & \frac{1}{T} \int_{r}^{T} \sup _{\tau \in[t-r, t]}\left(\int_{0}^{t-r} \phi(\tau-s)\|f(s+\omega)-f(s)\| d s\right) d t \\
& +\frac{1}{T} \int_{r}^{T} \sup _{\tau \in[t-r, t]}\left(\int_{t-r}^{\tau} \phi(\tau-s)\|f(s+\omega)-f(s)\| d s\right) d t \\
:= & J_{1}(T)+J_{2}(T),
\end{aligned}
$$

where

$$
\begin{aligned}
& J_{1}(T)=\frac{1}{T} \int_{r}^{T} \sup _{\tau \in[t-r, t]}\left(\int_{0}^{t-r} \phi(\tau-s)\|f(s+\omega)-f(s)\| d s\right) d t \\
& J_{2}(T)=\frac{1}{T} \int_{r}^{T} \sup _{\tau \in[t-r, t]}\left(\int_{t-r}^{\tau} \phi(\tau-s)\|f(s+\omega)-f(s)\| d s\right) d t .
\end{aligned}
$$


From Fubini's theorem and $\phi$ is nonincreasing, one has

$$
\begin{aligned}
J_{1}(T) & =\frac{1}{T} \int_{r}^{T}\left(\int_{0}^{t-r} \sup _{\tau \in[t-r, t]} \phi(\tau-s)\|f(s+\omega)-f(s)\| d s\right) d t \\
& \leq \frac{1}{T} \int_{r}^{T}\left(\int_{0}^{t-r} \phi(t-r-s)\|f(s+\omega)-f(s)\| d s\right) d t \\
& =\frac{1}{T} \int_{r}^{T}\left(\int_{0}^{t-r} \phi(s)\|f(t-r-s+\omega)-f(t-r-s)\| d s\right) d t \\
& =\frac{1}{T} \int_{r}^{T}\left(\int_{r}^{t} \phi(s-r)\|f(t-s+\omega)-f(t-s)\| d s\right) d t \\
& =\frac{1}{T} \int_{r}^{T} \phi(s-r)\left(\int_{s}^{T}\|f(t-s+\omega)-f(t-s)\| d t\right) d s \\
& =\int_{r}^{T} \phi(s-r)\left(\frac{1}{T} \int_{0}^{T-s}\|f(t+\omega)-f(t)\| d t\right) d s \\
& =\int_{r}^{T} \phi(s-r) \varphi_{T}(s) d s \\
& \leq \int_{r}^{T} \phi(s-r) \varphi_{T}(0) d s \\
& \leq \varphi_{T}(0)\|\varphi\|_{L^{1}} \rightarrow 0, \quad T \rightarrow \infty .
\end{aligned}
$$

By $f \in P \mathcal{S} A P_{\omega, r}\left(\mathbf{R}^{+}, X\right)$, one has

$$
\begin{aligned}
J_{2}(T) & =\frac{1}{T} \int_{r}^{T} \sup _{\tau \in[t-r, t]}\left(\int_{t-r}^{\tau} \phi(\tau-s)\|f(s+\omega)-f(s)\| d s\right) d t \\
& \leq \frac{1}{T} \int_{r}^{T} \phi(0) \sup _{\tau \in[t-r, t]}\left(\int_{t-r}^{\tau}\|f(s+\omega)-f(s)\| d s\right) d t \\
& \leq \frac{\phi(0)}{T} \int_{r}^{T}\left(\int_{t-r}^{t}\|f(s+\omega)-f(s)\| d s\right) d t \\
& \leq \frac{\phi(0)}{T} \int_{r}^{T}\left(\int_{t-r}^{t} \sup _{s \in[t-r, t]}\|f(s+\omega)-f(s)\| d s\right) d t \\
& \leq \frac{r \phi(0)}{T} \int_{r}^{T} \sup _{s \in[t-r, t]}\|f(s+\omega)-f(s)\| d t \rightarrow 0, \quad T \rightarrow \infty .
\end{aligned}
$$

So (3.6) holds. 
By (3.5), (3.6), one has

$$
\lim _{T \rightarrow \infty} \frac{1}{T} \int_{r}^{T} \sup _{\tau \in[t-r, t]}\|(\Lambda f)(\tau+\omega)-(\Lambda f)(\tau)\| d t=0,
$$

which means that $\Lambda f \in P \mathcal{S} A P_{\omega, r}\left(\mathbf{R}^{+}, X\right)$. The proof is complete.

\section{Fractional differential equations with delay}

In this section, we establish some sufficient criteria for the existence and uniqueness of $P \mathcal{S} A P_{\omega, r}$ solutions for (1.1).

We adopt the following concept of mild solution.

Definition 4.1 [14]. Suppose $0<\alpha \leq \beta \leq 1, \quad \mu>0$. A function $u \in$ $C([-r, \infty), X)$ is said to be a mild solution of $(1.1)$ if $u_{0}=\varphi_{1}, u_{0}^{\prime}=\varphi_{2}$ and for $t \in \mathbf{R}^{+}$,

$$
\begin{aligned}
u(t)= & S_{\alpha, \beta}(t) \varphi_{1}(0)+\left(g_{1} * S_{\alpha, \beta}\right)(t) \varphi_{2}(0) \\
& +\mu\left(g_{1+\alpha-\beta} * S_{\alpha, \beta}\right)(t) \varphi_{1}(0)+\int_{0}^{t} S_{\alpha, \beta}(t-s) f\left(s, u_{s}\right) d s .
\end{aligned}
$$

To establish our results, we introduce the following conditions:

$\left(H_{1}\right) \quad A$ is an $\tilde{\omega}$-sectorial operator of angle $\beta \pi / 2$ with $\tilde{\omega}<0$.

$\left(H_{2}\right) \quad f \in P \mathcal{S} A P_{\omega, r}\left(\mathbf{R}^{+} \times \mathscr{C}, X\right)$.

$\left(H_{31}\right) f$ satisfies the Lipschitz condition

$$
\|f(t, \phi)-f(t, \psi)\| \leq L_{f}\|\phi-\psi\|_{\mathscr{C}}, \quad \phi, \psi \in \mathscr{C}, t \in \mathbf{R}^{+} .
$$

where $L_{f}>0$ is a constant.

$\left(H_{32}\right) f$ satisfies the Lipschitz condition

$$
\|f(t, \phi)-f(t, \psi)\| \leq L_{f}(t)\|\phi-\psi\|_{\mathscr{C}}, \quad \phi, \psi \in \mathscr{C}, t \in \mathbf{R}^{+},
$$

where $L_{f} \in B S^{p}\left(\mathbf{R}^{+}, \mathbf{R}^{+}\right), p \geq 1$.

$\left(H_{33}\right) f$ satisfies the Lipschitz condition

$$
\|f(t, \phi)-f(t, \psi)\| \leq L_{f}(t)\|\phi-\psi\|_{\mathscr{C}}, \quad \phi, \psi \in \mathscr{C}, t \in \mathbf{R}^{+},
$$

where $L_{f} \in B S^{p}\left(\mathbf{R}^{+}, \mathbf{R}^{+}\right) \cap L^{1}\left(\mathbf{R}^{+}, \mathbf{R}^{+}\right), p \geq 1$.

Theorem 4.1. Assume that $\left(H_{1}\right),\left(H_{2}\right),\left(H_{31}\right)$ hold, then (1.1) has a unique mild solution $u(t) \in P \mathcal{S} A P_{\omega, r}\left(\mathbf{R}^{+}, X\right)$ if $C L_{f}<1$, where $C$ is the constant defined in Theorem 2.1.

Proof. By Theorem 2.1, $A$ generates a uniformly integrable $(\alpha, \beta)_{\mu^{-}}$ regularized family $S_{\alpha, \beta}(t)$ on Banach space $X$. Let $\mathfrak{B}=\left\{u:[-r, \infty) \rightarrow X \mid u_{0}\right.$ 
$\left.=\varphi_{1}, u_{0}^{\prime}=\varphi_{2},\left.u\right|_{[0, \infty)} \in P \mathcal{S} A P_{\omega, r}\left(\mathbf{R}^{+}, X\right)\right\}$ endowed with the metric $d(u, v)=$ $\|u-v\|_{C\left(\mathbf{R}^{+}, X\right)}$ and let $\mathscr{F}: \mathfrak{B} \rightarrow \mathfrak{B}$ be the map defined by $(\mathscr{F} u)_{0}=\varphi_{1}$, $(\mathscr{F} u)_{0}^{\prime}=\varphi_{2}$ and

$$
\begin{aligned}
(\mathscr{F} u)(t)= & S_{\alpha, \beta}(t) \varphi_{1}(0)+\left(g_{1} * S_{\alpha, \beta}\right)(t) \varphi_{2}(0)+\mu\left(g_{1+\alpha-\beta} * S_{\alpha, \beta}\right)(t) \varphi_{1}(0) \\
& +\int_{0}^{t} S_{\alpha, \beta}(t-s) f\left(s, u_{s}\right) d s, \quad t \in \mathbf{R}^{+} .
\end{aligned}
$$

Let $u \in \mathfrak{B}$ be given,

(i) $\lim _{t \rightarrow \infty}\left\|S_{\alpha, \beta}(t) \varphi_{1}(0)\right\|=0 \quad$ by $\quad(2.2), \quad$ so $\quad S_{\alpha, \beta}(t) \varphi_{1}(0) \in C_{0}\left(\mathbf{R}^{+}, X\right) \subset$ $P \mathcal{S} A P_{\omega, r}\left(\mathbf{R}^{+}, X\right)$.

(ii) By (2.2), one has $\sup _{t>\tau}\left\|t S_{\alpha, \beta}(t)\right\|<\infty$ for each $\tau>0$. Since $A$ is an $\tilde{\omega}$-sectorial of angle $\beta \pi / 2$, then $\left\|\hat{S}_{\alpha, \beta}(\lambda)\right\| \rightarrow 0$ as $\lambda \rightarrow 0$. Thus, by the Hardy-Littlewood theorem (Theorem 2.2), we conclude that $\lim _{t \rightarrow \infty}\left\|\left(g_{1} * S_{\alpha, \beta}\right)(t)\right\|=0$. Hence $\quad\left(g_{1} * S_{\alpha, \beta}\right)(t) \varphi_{2}(0) \in C_{0}\left(\mathbf{R}^{+}, X\right) \subset$ $P \mathcal{S A P}{ }_{\omega, r}\left(\mathbf{R}^{+}, X\right)$.

(iii) Let $0<\varepsilon<\beta-\alpha$, then

$$
\begin{aligned}
& \left\|g_{1+\alpha-\beta} * S_{\alpha, \beta}(t)\right\| \\
& \quad=\left\|\int_{0}^{t} g_{1+\alpha-\beta}(t-\tau) S_{\alpha, \beta}(\tau) d \tau\right\| \\
& \quad=\left\|\Gamma(\beta-\alpha-\varepsilon) \int_{0}^{t} g_{1+\alpha-\beta}(t-\tau) g_{\beta-\alpha-\varepsilon}(\tau) \tau^{\alpha-\beta+\varepsilon+1} S_{\alpha, \beta}(\tau) d \tau\right\| \\
& \quad \leq \Gamma(\beta-\alpha-\varepsilon) \int_{0}^{t} g_{1+\alpha-\beta}(t-\tau) g_{\beta-\alpha-\varepsilon}(\tau) \tau^{\alpha-\beta+\varepsilon+1}\left\|S_{\alpha, \beta}(\tau)\right\| d \tau,
\end{aligned}
$$

By (2.2), one has

$$
\Gamma(\beta-\alpha-\varepsilon) \tau^{\alpha-\beta+\varepsilon+1}\left\|S_{\alpha, \beta}(\tau)\right\| \leq \frac{\tilde{M} \tau^{\alpha-\beta+\varepsilon+1}}{1+|\tilde{\omega}| \tau^{\alpha+1}}=\frac{\tilde{M} \tau^{-\beta+\varepsilon}}{\frac{1}{\tau^{\alpha+1}}+|\tilde{\omega}|}, \quad \tau>0,
$$

where $\tilde{M}$ is a constant. Since $\varepsilon<\beta$, there exists a constant $\tilde{C}>0$ such that

$$
\Gamma(\beta-\alpha-\varepsilon) \tau^{\alpha-\beta+\varepsilon+1}\left\|S_{\alpha, \beta}(\tau)\right\| \leq \tilde{C} .
$$

Therefore,

$$
\begin{aligned}
\left\|g_{1+\alpha-\beta} * S_{\alpha, \beta}(t)\right\| & \leq \tilde{C} \int_{0}^{t} g_{1+\alpha-\beta}(t-\tau) g_{\beta-\alpha-\varepsilon}(\tau) d \tau \\
& =\tilde{C} g_{1-\varepsilon}(t)=\frac{\tilde{C} t^{-\varepsilon}}{\Gamma(1-\varepsilon)}
\end{aligned}
$$

which shows that $\left\|g_{1+\alpha-\beta} * S_{\alpha, \beta}(t)\right\| \rightarrow 0$ as $t \rightarrow \infty$. Therefore, $g_{1+\alpha-\beta} *$ $S_{\alpha, \beta} \in P \mathcal{S} A P_{\omega, r}\left(\mathbf{R}^{+}, X\right)$. 
(iv) For $u \in P \mathcal{S} A P_{\omega, r}\left(\mathbf{R}^{+}, X\right), u_{s} \in P \mathcal{S} A P_{\omega, r}\left(\mathbf{R}^{+}, \mathscr{C}\right)$ by Lemma 3.5. It is not difficult to see that $f\left(s, u_{s}\right) \in P \mathcal{S} A P_{\omega, r}\left(\mathbf{R}^{+}, X\right)$ by Theorem 3.1. By Theorem 2.1, Lemma 3.6, one has

$$
\int_{0}^{t} S_{\alpha, \beta}(t-s) f\left(s, u_{s}\right) d s \in P \mathcal{S} A P_{\omega, r}\left(\mathbf{R}^{+}, X\right) .
$$

By (i)-(iv), $\mathscr{F} u \in \mathfrak{B}$, so $\mathscr{F}$ is well defined.

Moreover, let $u, v \in \mathfrak{B}$, one has

$$
\begin{aligned}
\|(\mathscr{F} u)(t)-(\mathscr{F} v)(t)\| & \leq \int_{0}^{t}\left\|S_{\alpha, \beta}(t-s)\right\|\left\|f\left(s, u_{s}\right)-f\left(s, v_{s}\right)\right\| d s \\
& \leq L_{f}\|u-v\| \cdot \int_{0}^{t} \frac{C}{1+|\tilde{\omega}|\left[(t-s)^{\alpha+1}+\mu(t-s)^{\beta}\right]} d s \\
& \leq C L_{f}\|u-v\|,
\end{aligned}
$$

which implies that

$$
d(\mathscr{F} u, \mathscr{F} v) \leq C L_{f} d(u, v) .
$$

By the Banach contraction mapping principle, $\mathscr{F}$ has a unique fixed point in $\mathfrak{B}$, which is the unique $P \mathcal{S} A P_{\omega, r}$ mild solution of (1.1).

THEOREM 4.2. Assume that $\left(H_{1}\right),\left(H_{2}\right),\left(H_{32}\right)$ hold and

$$
C\left(1+\frac{|\tilde{\omega}|^{-1 /(\alpha+1)} \pi}{(\alpha+1) \sin (\pi /(\alpha+1))}\right)\left\|L_{f}\right\|_{S^{p}}<1,
$$

then (1.1) has a unique mild solution $u(t) \in P \mathcal{S} A P_{\omega, r}\left(\mathbf{R}^{+}, X\right)$.

Proof. Define the operator $\mathscr{F}$ as in (4.2), it is easy to see that $f\left(s, u_{s}\right) \in$ $P \mathcal{S} A P_{\omega, r}\left(\mathbf{R}^{+}, X\right)$ by Theorem 3.2 and $\mathscr{F}$ is well defined similarly as the proof of Theorem 4.1.

For $u, v \in \mathfrak{B}$, one has

$$
\begin{aligned}
\|(\mathscr{F} u)(t)-(\mathscr{F} v)(t)\| & \leq \int_{0}^{t}\left\|S_{\alpha, \beta}(t-s)\right\|\left\|f\left(s, u_{s}\right)-f\left(s, v_{s}\right)\right\| d s \\
& \leq\|u-v\| \cdot \int_{0}^{t} \frac{C L_{f}(s)}{1+|\tilde{\omega}|\left[(t-s)^{\alpha+1}+\mu(t-s)^{\beta}\right]} d s \\
& \leq\|u-v\| \cdot \int_{0}^{t} \frac{C L_{f}(s)}{1+|\tilde{\omega}|(t-s)^{\alpha+1}} d s,
\end{aligned}
$$

- If $t=m \in \mathbf{N}$, in this case 


$$
\begin{aligned}
& \int_{0}^{t} \frac{1}{1+|\tilde{\omega}|(t-s)^{\alpha+1}} L_{f}(s) d s \\
& =\int_{0}^{m} \frac{1}{1+|\tilde{\omega}|(m-s)^{\alpha+1}} L_{f}(s) d s \\
& =\sum_{k=0}^{m-1} \int_{k}^{k+1} \frac{1}{1+|\tilde{\omega}|(m-s)^{\alpha+1}} L_{f}(s) d s \\
& \leq \sum_{k=0}^{m-1} \frac{1}{1+|\tilde{\omega}|(m-k-1)^{\alpha+1}} \int_{k}^{k+1} L_{f}(s) d s \\
& \leq \sum_{k=0}^{m-1} \frac{1}{1+|\tilde{\omega}|(m-k-1)^{\alpha+1}}\left(\int_{k}^{k+1} L_{f}(s)^{p} d s\right)^{1 / p} \\
& \leq\left[1+\left(\int_{0}^{1}+\int_{1}^{2}+\cdots+\int_{m-2}^{m-1}\right) \frac{1}{1+|\tilde{\omega}| t^{\alpha+1}} d t\right]\left\|L_{f}\right\|_{S^{p}} \\
& \leq\left(1+\int_{0}^{\infty} \frac{1}{1+|\tilde{\omega}| t^{\alpha+1}} d t\right)\left\|L_{f}\right\|_{S^{p}} \\
& \leq\left(1+\frac{|\tilde{\omega}|^{-1 /(\alpha+1)} \pi}{(\alpha+1) \sin (\pi /(\alpha+1))}\right)\left\|L_{f}\right\|_{S^{p}},
\end{aligned}
$$

- If $t=m-h$, where $0<h<1$. In this general case,

$$
\begin{aligned}
\int_{0}^{t} \frac{1}{1+|\tilde{\omega}|(t-s)^{\alpha+1}} L_{f}(s) d s & =\int_{0}^{m-h} \frac{1}{1+|\tilde{\omega}|(m-h-s)^{\alpha+1}} L_{f}(s) d s \\
& =\int_{h}^{m} \frac{1}{1+|\tilde{\omega}|(m-s)^{\alpha+1}} L_{f}(s-h) d s \\
& =\int_{0}^{m} \frac{1}{1+|\tilde{\omega}|(m-s)^{\alpha+1}} \tilde{L}_{f}(s) d s \\
& \leq\left(1+\frac{|\tilde{\omega}|^{-1 /(\alpha+1)} \pi}{(\alpha+1) \sin (\pi /(\alpha+1))}\right)\left\|\tilde{L}_{f}\right\|_{S^{p}},
\end{aligned}
$$

where $\tilde{L}_{f}$ is defined by

$$
\tilde{L}_{f}(s)= \begin{cases}0, & 0 \leq s<h \\ L_{f}(s-h), & s \geq h\end{cases}
$$

then $\left\|\tilde{L}_{f}\right\|_{S^{p}}=\left\|L_{f}\right\|_{S^{p}}$. So we infer that

$$
\int_{0}^{t} \frac{1}{1+|\tilde{\omega}|(t-s)^{\alpha+1}} L_{f}(s) d s \leq\left(1+\frac{|\tilde{\omega}|^{-1 /(\alpha+1)} \pi}{(\alpha+1) \sin (\pi /(\alpha+1))}\right)\left\|L_{f}\right\|_{S^{p}}
$$


By (4.3), (4.4), one has

$$
\|(\mathscr{F} u)(t)-(\mathscr{F} v)(t)\| \leq C\left(1+\frac{|\tilde{\omega}|^{-1 /(\alpha+1)} \pi}{(\alpha+1) \sin (\pi /(\alpha+1))}\right)\left\|L_{f}\right\|_{S^{p}} \cdot\|u-v\|,
$$

so

$$
d(\mathscr{F} u, \mathscr{F} v) \leq C\left(1+\frac{|\tilde{\omega}|^{-1 /(\alpha+1)} \pi}{(\alpha+1) \sin (\pi /(\alpha+1))}\right)\left\|L_{f}\right\|_{S^{p}} \cdot d(u, v) .
$$

By the Banach contraction mapping principle, $\mathscr{F}$ has a unique fixed point in $\mathfrak{B}$, which is the unique $P \mathcal{S} A P_{\omega, r}$ mild solution of (1.1).

THEOREM 4.3. Assume that $\left(H_{1}\right),\left(H_{2}\right),\left(H_{33}\right)$ hold, then $(1.1)$ has a unique mild solution $u(t) \in P \mathcal{S} A P_{\omega, r}\left(\mathbf{R}^{+}, X\right)$.

Proof. Define the operator $\mathscr{F}$ as in (4.2). Let $u, v \in \mathfrak{B}$, one has

$$
\begin{aligned}
\|(\mathscr{F} u)(t)-(\mathscr{F} v)(t)\| & \leq \int_{0}^{t}\left\|S_{\alpha, \beta}(t-s)\right\|\left\|f\left(s, u_{s}\right)-f\left(s, v_{s}\right)\right\| d s \\
& \leq\|u-v\| \cdot \int_{0}^{t} \frac{C L_{f}(s)}{1+|\tilde{\omega}|\left[(t-s)^{\alpha+1}+\mu(t-s)^{\beta}\right]} d s \\
& \leq C \int_{0}^{t} L_{f}(s) d s \cdot\|u-v\| \\
& \leq C\left\|L_{f}\right\|_{L^{1}} \cdot\|u-v\| .
\end{aligned}
$$

Similarly,

$$
\begin{aligned}
\left\|\left(\mathscr{F}^{2} u\right)(t)-\left(\mathscr{F}^{2} v\right)(t)\right\| & \leq \int_{0}^{t} \frac{C L_{f}(s)}{1+|\tilde{\omega}|\left[(t-s)^{\alpha+1}+\mu(t-s)^{\beta}\right]}\|(\mathscr{F} u)(s)-(\mathscr{F} v)(s)\| d s \\
& \leq C \int_{0}^{t} L_{f}(s)\|(\mathscr{F} u)(s)-(\mathscr{F} v)(s)\| d s \\
& \leq C^{2}\|u-v\| \int_{0}^{t} L_{f}(s)\left(\int_{0}^{s} L_{f}(\tau) d \tau\right) d s \\
& =C^{2}\|u-v\| \int_{0}^{t}\left(\int_{0}^{s} L_{f}(\tau) d \tau\right) d\left(\int_{0}^{s} L_{f}(\tau) d \tau\right) \\
& \leq \frac{C^{2}}{2 !}\|u-v\|\left(\int_{0}^{t} L_{f}(\tau) d \tau\right)^{2} \\
& \leq \frac{\left(C\left\|L_{f}\right\|_{L^{1}}\right)^{2}}{2 !}\|u-v\| .
\end{aligned}
$$


By the method of mathematical induction, we have

$$
\left\|\left(\mathscr{F}^{n} u\right)(t)-\left(\mathscr{F}^{n} v\right)(t)\right\| \leq \frac{C^{n}}{n !}\|u-v\|\left(\int_{0}^{t} L_{f}(\tau) d \tau\right)^{n} .
$$

Moreover, since $L_{f}(t) \in L^{1}\left(\mathbf{R}^{+}, \mathbf{R}^{+}\right)$,

$$
\left\|\left(\mathscr{F}^{n} u\right)(t)-\left(\mathscr{F}^{n} v\right)(t)\right\| \leq \frac{\left(C\left\|L_{f}\right\|_{L^{1}}\right)^{n}}{n !}\|u-v\|,
$$

which implies that

$$
d\left(\mathscr{F}^{n} u, \mathscr{F}^{n} v\right) \leq \frac{\left(C\left\|L_{f}\right\|_{L^{1}}\right)^{n}}{n !} d(u, v)
$$

For sufficiently large $n$, we have $\left(C\left\|L_{f}\right\|_{L^{1}}\right)^{n} / n !<1$, by the Banach contraction mapping principle, $\mathscr{F}$ has a unique fixed point in $\mathfrak{B}$, which is the unique $P \mathcal{S} A P_{\omega, r}$ mild solution of (1.1).

\section{Example}

Consider the following fractional partial differential equation with delay

$$
\begin{array}{rl}
D_{t}^{\alpha+1} & u(t, x)+\mu D_{t}^{\beta} u(t, x) \\
& =\frac{\partial^{2}}{\partial x^{2}} u(t, x)-\delta u(t, x)+a(t) \int_{-1}^{0} b(s) \sin [u(t+s, x)] d s,
\end{array}
$$

where $t \in \mathbf{R}^{+}, x \in[0,1], 0<\alpha \leq \beta \leq 1, \mu>0, \delta>0, a(t) \in P \mathcal{S} A P_{\omega, 1}\left(\mathbf{R}^{+}, \mathbf{R}\right)$, with initial and zero boundary conditions.

Let $X=\left(L^{2}([0,1], \mathbf{R}),\|\cdot\|_{L^{2}}\right)$ and define the operator $A$ on $X$ by

$$
A u=\frac{\partial^{2}}{\partial x^{2}} u-\delta u
$$

with

$$
D(A)=\left\{u \in L^{2}([0,1], \mathbf{R}): u^{\prime \prime} \in L^{2}[0,1], u(0)=u(1)=0\right\}
$$

and

$$
f(t, \varphi)(x)=a(t) \int_{-1}^{0} b(s) \sin [\varphi(s)(x)] d s, \quad t \in \mathbf{R}^{+}, \varphi \in C([-1,0], X), x \in[0,1] .
$$

It is well know that $A$ is a $\tilde{\omega}$-sectorial operator with $\tilde{\omega}=-\delta<0$ and angle $\pi / 2$ (and hence of angle $\beta \pi / 2$ with $\beta \leq 1$ ) [14]. (5.1) can be rewritten as an abstract system of the form (1.1), where $u(t)=u(t, \cdot)$. In addition, since $a(t) \in$ $P \mathcal{S} A P_{\omega, 1}\left(\mathbf{R}^{+}, \mathbf{R}\right)$ and

$\|f(t, \phi)-f(t, \psi)\| \leq\|a\|\left(\int_{-1}^{0}|b(s)|^{2} d s\right)^{1 / 2}\|\phi-\psi\|_{\mathscr{C}}, \quad$ for all $\phi, \psi \in C([-1,0], X)$, 
so $\left(H_{31}\right)$ holds with $L_{f} \equiv\|a\|\left(\int_{-1}^{0}|b(s)|^{2} d s\right)^{1 / 2}$. By Theorem 4.1, we conclude that (5.1) has a unique solution $u \in P \mathcal{S} A P_{\omega, 1}$ if $C L_{f}<1$.

\section{REFERENCES}

[ 1 ] E. Alvarez-Pardo and C. Lizama, Pseudo asymptotic solutions of fractional order semilinear equations, Banach J. Math. Anal. 7 (2013), 42-52.

[2] W. Arendt, C. Batty, M. Hieber and F. Neubrander, Vector-valued Laplace transforms and Cauchy problems, Monographis in mathematics 96, Birkhäuser, Basel, 2001.

[3] J. Blot, P. Cieutat and G. M. N'GuéréKata, $\mathcal{S}$-asymptocially $\omega$-periodic functins and applications to evolution equations, Afr. Diaspora J. Math. 12 (2011), 113-121.

[ 4 ] C. Cuevas and J. C. De Souza, $\mathcal{S}$-asymptocially $\omega$-periodic solutions of semilinear fractional integro-differential equations, Appl. Math. Lett. 22 (2009), 865-870.

[5] C. Cuevas and C. Lizama, $\mathcal{S}$-asymptotically $\omega$-periodic solutions for semilinear Volterra equations, Math. Methods Appl. Sci. 33 (2010), 1628-1636.

[6] C. Cuevas and C. Lizama, Existence for $\mathcal{S}$-asymptotically $\omega$-periodic solutions for two-times fractional order differential equations, Southeast Asian Bull. Math. 37 (2013), 683-690.

[7] J. M. Cushing, Forced asymptotically periodic solutions of predator-prey systems with or without hereditary effects, SIAM J. Appl. Math. 30 (1976), 665-674.

[8] B. De Andrade and C. Cuevas, $\mathcal{S}$-asymptotically $\omega$-periodic and asymptotically $\omega$-periodic solutions to semi-linear Cauchy problems with non-dense domain, Nonlinear Anal. 72 (2010), 3190-3208.

[9] W. Dimbour ANd G. M. N'GuéréKATA, $\mathcal{S}$-asymptotically $\omega$-periodic solutions to some classes of partial evolution equations, Appl. Math. Comput. 218 (2012), 7622-7628.

[10] H. R. Henríquez, M. Pierri and P. TÁboas, On $\mathcal{S}$-asymptotically $\omega$-periodic functions on Banach spaces and applications, J. Math. Anal. Appl. 343 (2008), 1119-1130.

[11] H. R. Henríquez, M. Pierri and P. TÁboas, Existence of $\mathcal{S}$-asymptotically $\omega$-periodic solutions for abstract neutral functional-differential equations, Bull. Aust. Math. Soc. 78 (2008), 365-382.

[12] H. R. Henríquez, Asymptotically periodic solutions of abstract differential equations, Nonlinear Anal. 80 (2013), 135-149.

[13] H. R. Henríquez, C. Cuevas and A. Caicedo, Asymptotically periodic solutions of neutral partial differential equations with infinite delay, Commun. Pure Appl. Anal. 12 (2013), 2031-2068.

[14] V. Keyantuo, C. Lizama And M. Warma, Asymptotic behavior of fractional order semilinear evolution equations, Differential Integral Equations 26 (2013), 757-780.

[15] C. Lizama, An operator theoretical approach to a class of fractional order differential equations, Appl. Math. Lett. 24 (2011), 184-190.

[16] S. H. J. Nicola and M. Pierri, A note on $\mathcal{S}$-asymptotically $\omega$-periodic functions, Nonlinear Anal. Real World Appl. 10 (2009), 2937-2938.

[17] A. Pankov, Bounded and almost periodic solutions of nonlinear operator differential equations, Kluwer, Dordrecht, 1990.

[18] M. Pierri, On $\mathcal{S}$-asymptotically $\omega$-periodic functions and applications, Nonlinear Anal. 75 (2012), 651-661.

[19] M. Pierri AND V. Rolnik, On pseudo $\mathcal{S}$-asymptotically periodic functions, Bull. Aust. Math. Soc. 87 (2013), 238-254.

[20] M. Stojanović and R. Gorenflo, Nonlinear two-term time fractional diffusion-wave problem, Nonliear Anal. Real World Appl. 11 (2010), 3512-3523. 
[21] F. Y. Wei AND K. WANG, Asymptotically periodic logistic equation, J. Biomath. 20 (2005), 399-405.

[22] F. Y. WeI AND K. WANG, Global stability and asymptotically periodic solutions for nonautonomous cooperative Lotka-Volterra diffusion system, Appl. Math. Comput. 182 (2006), $161-165$.

[23] Z. J. ZENG, Asymptotically periodic solution and optimal harvesting policy for Gompertz system, Nonlinear Anal. Real World Appl. 12 (2011), 1401-1409.

Zhinan Xia

Department of Applied Mathematics

ZhEJIANG UNIVERSITY OF TECHNOLOGY

HANGZHou, ZheJiang, 310023

P.R. ChINA

E-mail: xiazn299@zjut.edu.cn 